DCPT-03/07

\title{
Strings in de Sitter space
}

\author{
Eugênio R. Bezerra de Mello* \\ Departamento de Física-CCEN, Universidade Federal da Paraíba, 58.059-970, J. Pessoa, PB, C. \\ Postal 5.008, Brazil \\ Yves Brihaye ${ }^{\dagger}$ \\ Faculté des Sciences, Université de Mons-Hainaut, B-7000 Mons, Belgium \\ Betti Hartmann ${ }^{\ddagger}$ \\ Department of Mathematical Sciences, University of Durham, Durham DH1 3LE, U.K.
}

(October 28, 2018)

\begin{abstract}
We study both global as well as local (Nielsen-Olesen) strings in de Sitter space. While these type of topological defects have been studied in the background of a de Sitter metric previously, we study here the full set of coupled equations. We find only "closed" solutions. The behaviour of the metric tensor of these solutions resembles that of "supermassive" strings with a curvature singularity at the cosmological horizon. For global strings (and the composite defect) we are able to construct solutions which are regular on the interval from the origin to the cosmological horizon if the global string core lies completely inside the horizon.
\end{abstract}

PACS numbers: 04.20.Jb, 04.40.Nr, 11.27.+d

*emello@fisica.ufpb.br

†Yves.Brihaye@umh.ac.be

‡Betti.Hartmann@durham.ac.uk 


\section{INTRODUCTION}

A number of different topological defects [1] are thought to have been formed during the phase transitions in the early universe. Depending on the topology of the vacuum manifold $\mathcal{M}$ these are domain walls, strings, monopoles and textures corresponding to the homotopy groups $\pi_{0}(\mathcal{M}), \pi_{1}(\mathcal{M}), \pi_{2}(\mathcal{M})$ and $\pi_{3}(\mathcal{M})$, respectively. Cosmic strings [1,2] have always gained a lot of interest since they are thought to be important for the structure formation

in the universe due to their huge energy per unit length (roughly $10^{21} \frac{\mathrm{kg}}{\mathrm{m}}$ for a string formed at GUT scale $\approx 10^{16} \mathrm{GeV}$ ).

A classical field theory model which has string-like solutions is the Abelian Higgs model [3]. These solutions, also sometimes called "vortices", correspond to infinitely long objects. They have a core radius inverse proportional to the Higgs boson mass and magnetic flux tubes with radius proportional to the inverse of the gauge boson mass. Coupling the Abelian Higgs model minimally to gravity, the influence of the vortex on the geometry of space-time was investigated analytically [4]. It was shown that far away from the core of the string the space-time is Minkowski minus a wedge. It was also realised [5] that if the vacuum expectation value of the Higgs field is sufficiently large (corresponding to strings having formed at a phase transition with energy scale much higher than the GUT scale), then a different type of solution is possible. These so-called "supermassive strings" exist only on a finite interval of the radial coordinate and have a curvature singularity at the maximal value of the radial coordinate. The existence of further solutions was investigated in a detailed numerical analysis [6,7]. It was found that the parameter space is indeed divided by the curve of maximal angular deficit $2 \pi$. If the deficit is smaller than $2 \pi$, so-called "open", i.e. infinitely extended solutions were found. One is the above mentioned cosmic string solution [4] which however has a "shadow" solution of Melvin-type for all values of the coupling constants. For deficit large than $2 \pi$, only the "supermassive", "closed" solutions exist [5].

The static solutions of the model without gauge field, so-called global strings, have also been studied [8-11]. Like all global defects, the global string has a long-range Goldstone field which leads to a divergent energy. Moreover, the global string is characterised by a logarithmically divergent deficit angle in contrast to the local string which has a constant deficit angle. The coupling to gravity in the case of the static global monopole leads to a singularity-free monopole solution [12] in the sense that while the energy is still linearly divergent, the solid deficit angle is now finite. For the static global string the corresponding singularity can not be removed by coupling the system to gravity and only the assumption that the metric be time-dependent removes the singularity [13].

Since a number of astrophysical observations like e.g. the measurement of redshifts of Type Ia supernovae [14] has led scientists to believe that we live in a universe with positive cosmological constant, the study of topological defects in de Sitter (dS) space seems interesting. But it also is of interest from another point of view, namely the dS/CFT correspondence [15]. This correspondence suggests a holographic duality between gravity in a $d$-dimensional dS space and a conformal field theory (CFT) "living" on the boundary of the dS spacetime and thus being $d$-1-dimensional.

Recently, Nielsen-Olesen strings in the background of a 4-dimensional de Sitter spacetime $d S_{4}$ have been studied [16]. However, to our knowledge, the full system of coupled matter and metric field equations has not been studied yet. One of the aims of this paper is the 
investigation of exactly this point.

Motivated by some recent work on a composite system of a global and local monopole in curved space-time [17-19], we investigate the composite system of a global and NielsenOlesen string in a curved space-time with cosmological constant as well.

Our paper is organised as follows: we give the model and static, cylindrically symmetric Ansatz in Section 2. We give the equations of motion in Section 3. We discuss the pure Nielsen-Olesen solutions in Section 4, the global string solutions in Section 5 and the composite system of a global and Nielsen-Olesen string in Section 6. We give our summary in Section 7.

\section{THE MODEL}

The model which describes a gravitating Nielsen-Olesen string interacting with a global one in the presence of a non-vanishing cosmological constant is given by the following action:

$$
S=\int d^{4} x \sqrt{-g}\left(\frac{1}{16 \pi G}(R-2 \Lambda)+\mathcal{L}_{N O}+\mathcal{L}_{\text {global }}+\mathcal{L}_{\text {inter }}\right)
$$

where $R$ is the Ricci scalar, $G$ denotes Newton's constant and $\Lambda$ is the cosmological constant. The Lagrangian of the Abelian Higgs model is given by [3]:

$$
\mathcal{L}_{N O}=\frac{1}{2} D_{\mu} \phi\left(D^{\mu} \phi\right)^{*}-\frac{1}{4} F_{\mu \nu} F^{\mu \nu}-\frac{\lambda_{1}}{4}\left(\phi \phi^{*}-\eta_{1}^{2}\right)^{2}
$$

with the covariant derivative $D_{\mu}=\nabla_{\mu}-i e A_{\mu}$ and the field strength $F_{\mu \nu}=\partial_{\mu} A_{\nu}-\partial_{\nu} A_{\mu}$ of the $\mathrm{U}(1)$ gauge potential $A_{\mu}$ with coupling constant $e . \phi$ is a complex scalar field (the Higgs field) with vacuum expectation value $\eta_{1}$ and self-coupling constant $\lambda_{1}$. The Lagrangian of the global string reads [1]:

$$
\mathcal{L}_{\text {global }}=\frac{1}{2} \partial_{\mu} \chi \partial^{\mu} \chi^{*}-\frac{\lambda_{2}}{4}\left(\chi \chi^{*}-\eta_{2}^{2}\right)^{2}
$$

where $\chi$ is a complex scalar field (the Goldstone field) with vacuum expectation value $\eta_{2}$ and self-coupling $\lambda_{2}$. Finally, following [17] we introduce an extra potential which couples (with coupling constant $\lambda_{3}$ ) the two sectors of the model directly to each other:

$$
\mathcal{L}_{\text {inter }}=-\frac{\lambda_{3}}{4}\left(\phi \phi^{*}-\eta_{1}^{2}\right)\left(\chi \chi^{*}-\eta_{2}^{2}\right)
$$

Without this term, the global and local string would be coupled only indirectly over gravity. In this paper we will use units which $\hbar=c=1$.

\section{A. The Ansatz}

In the following we shall analyse the classical equations of motion associated with the above system. In order to do that, let us write down the matter and gravitational fields as

shown below. The most general, cylindrically symmetric line element invariant under boosts along the $z$-direction is: 


$$
d s^{2}=N^{2}(\rho) d t^{2}-d \rho^{2}-L^{2}(\rho) d \varphi^{2}-N^{2}(\rho) d z^{2} .
$$

The non-vanishing components of the Einstein tensor $G_{\mu \nu}$ then read:

$$
\begin{aligned}
& G_{t t}=-G_{z z}=\frac{N}{L}\left(L \partial_{\rho \rho} N+\partial_{\rho} N \partial_{\rho} L+N \partial_{\rho \rho} L\right) \\
& G_{r r}=\frac{\partial_{\rho} N}{N^{2} L}\left(2 \partial_{\rho} L N+\partial_{\rho} N L\right) \quad, \quad G_{\varphi \varphi}=\frac{L^{2}}{N^{2}}\left(2 N \partial_{\rho \rho} N+\left(\partial_{\rho} N\right)^{2}\right)
\end{aligned}
$$

where $\partial_{\rho}$ denotes the derivative with respect to $\rho$.

For the matter and gauge fields, we have:

$$
\begin{gathered}
\phi(\rho, \varphi)=\eta_{1} h(\rho) e^{i n \varphi}, \\
\chi(\rho, \varphi)=\eta_{1} f(\rho) e^{i m \varphi}, \\
A_{\mu} d x^{\mu}=\frac{1}{e}(n-P(\rho)) d \varphi .
\end{gathered}
$$

$n$ and $m$ are integers indexing the vorticity of the Higgs and Goldstone fields, respectively, around the $z$-axis.

Substituting the above configurations into the matter Lagrangian density $\mathcal{L}_{M}=\mathcal{L}_{N O}+$ $\mathcal{L}_{\text {global }}+\mathcal{L}_{\text {inter }}$, we obtain:

$$
\begin{aligned}
\mathcal{L}_{M} & =-\frac{\eta_{1}^{2}}{2}\left(\partial_{\rho} h(\rho)\right)^{2}-\frac{\eta_{1}^{2}}{2}\left(\partial_{\rho} f(\rho)\right)^{2}-\frac{n^{2}}{2 e^{2} L^{2}(\rho)}\left(\partial_{\rho} P(\rho)\right)^{2} \\
& -\frac{\eta_{1}^{2} n^{2}}{2 L^{2}(\rho)} h^{2}(\rho) P^{2}(\rho)-\frac{\eta_{1}^{2} m^{2}}{2 L^{2}(\rho)} f^{2}(\rho)-\frac{\lambda_{1} \eta_{1}^{4}}{4}\left(h^{2}(\rho)-1\right)^{2} \\
& -\frac{\lambda_{2} \eta_{1}^{4}}{4}\left(f^{2}(\rho)-q^{2}\right)^{2}-\frac{\lambda_{3} \eta_{1}^{4}}{2}\left(h^{2}(\rho)-1\right)\left(f^{2}(\rho)-q^{2}\right)
\end{aligned}
$$

\section{EQUATIONS OF MOTION}

We define the following dimensionless variable and function:

$$
x=\sqrt{\lambda_{1}} \eta_{1} \rho \quad, \quad L(x)=L(\rho) \eta_{1} \sqrt{\lambda_{1}} .
$$

Then, the total Lagrangian only depends on the following dimensionless coupling constants

$$
\gamma=8 \pi G \eta_{1}^{2} \quad, \alpha=e^{2} / \lambda_{1} \quad, \quad q=\frac{\eta_{2}}{\eta_{1}}, \bar{\Lambda}=\frac{\Lambda}{\lambda_{1} \eta_{1}^{2}} \quad, \quad \beta_{i}^{2}=\frac{\lambda_{i}}{\lambda_{1}} \quad, \quad i=1,2,3 .
$$

Varying (1) with respect to the matter fields and metric functions, we obtain a system of five non-linear differential equations. The Euler-Lagrange equations for the matter field functions read: 


$$
\begin{gathered}
\frac{\left(N^{2} L h^{\prime}\right)^{\prime}}{N^{2} L}=\frac{n^{2}}{L^{2}} h P^{2}+h\left(h^{2}-1\right)+\beta_{3}^{2} h\left(f^{2}-q^{2}\right), \\
\frac{\left(N^{2} L f^{\prime}\right)^{\prime}}{N^{2} L}=\frac{m^{2} f}{L^{2}}+\beta_{2}^{2} f\left(f^{2}-q^{2}\right)+\beta_{3}^{2} f\left(h^{2}-1\right), \\
\frac{L}{N^{2}}\left(\frac{N^{2} P^{\prime}}{L}\right)^{\prime}=\alpha h^{2} P,
\end{gathered}
$$

while the Einstein equations

$$
G_{\mu \nu}+\bar{\Lambda} g_{\mu \nu}=\gamma T_{\mu \nu} \quad, \quad \mu, \nu=t, x, \varphi, z
$$

read:

$$
\begin{aligned}
\frac{\left(L N N^{\prime}\right)^{\prime}}{N^{2} L} & =-\bar{\Lambda}+\gamma\left[\frac{n^{2}\left(P^{\prime}(x)\right)^{2}}{2 \alpha L^{2}}-\frac{1}{4}\left(h^{2}(x)-1\right)^{2}-\frac{\beta_{2}^{2}}{4}\left(f^{2}(x)-q^{2}\right)^{2}\right. \\
& \left.-\frac{\beta_{3}^{2}}{2}\left(h^{2}(x)-1\right)\left(f^{2}(x)-q^{2}\right)\right]
\end{aligned}
$$

and

$$
\begin{aligned}
\frac{\left(N^{2} L^{\prime}\right)^{\prime}}{N^{2} L} & =-\bar{\Lambda}-\gamma\left[\frac{n^{2} h^{2}(x) P^{2}(x)}{L^{2}(x)}+\frac{m^{2} f^{2}(x)}{L^{2}(x)}+\frac{n^{2}\left(P^{\prime}(x)\right)^{2}}{2 \alpha L^{2}(x)}+\frac{1}{4}\left(h^{2}(x)-1\right)^{2}\right. \\
& \left.+\frac{\beta_{2}^{2}}{4}\left(f^{2}(x)-q^{2}\right)^{2}+\frac{\beta_{3}^{2}}{2}\left(h^{2}(x)-1\right)\left(f^{2}(x)-q^{2}\right)\right]
\end{aligned}
$$

Moreover, defining $u=\sqrt{-g}=N^{2} L$ we get the following equation:

$$
\begin{aligned}
\frac{u^{\prime \prime}(x)}{u(x)} & =-3 \bar{\Lambda}-\gamma\left[\frac{n^{2} h^{2}(x) P^{2}(x)}{L^{2}(x)}+\frac{m^{2} f^{2}(x)}{L^{2}(x)}-\frac{n^{2}\left(P^{\prime}(x)\right)^{2}}{2 \alpha L^{2}(x)}\right. \\
& +\frac{3}{4}\left(h^{2}(x)-1\right)^{2}+\frac{3 \beta_{2}^{2}}{4}\left(f^{2}(x)-q^{2}\right)^{2} \\
& \left.+\frac{3 \beta_{3}^{2}}{2}\left(h^{2}(x)-1\right)\left(f^{2}(x)-q^{2}\right)\right] .
\end{aligned}
$$

The prime now denotes the derivatives with respect to $x$.

\section{A. Boundary Conditions}

The requirement of regularity at the origin leads to the following boundary conditions:

$$
h(0)=0, f(0)=0, P(0)=n
$$

for the matter fields and 


$$
N(0)=1, N^{\prime}(0)=0, L(0)=0, L^{\prime}(0)=1
$$

for the metric fields. Since a cosmological horizon appears naturally in de Sitter space, we integrate the equations only up to this value of the coordinate $x, x=x_{0}$. In order for the core of the local string to lie completely within the horizon we require:

$$
h\left(x=x_{0}\right)=1, f\left(x=x_{0}\right)=q, P\left(x=x_{0}\right)=0 .
$$

Note that due to the fact that the $x$ interval is finite, this is not (like in asymptotically flat space) a necessary condition for finite energy solutions. However, we have chosen these boundary conditions such that the energy-momentum tensor vanishes at $x=x_{0}$. In addition, the limit $\bar{\Lambda} \rightarrow 0$ which leads to $x_{0} \rightarrow \infty$ can be taken with these boundary conditions.

\section{NIELSEN-OLESEN STRINGS IN DE SITTER SPACE}

First, we are interested in the case of the pure Nielsen-Olesen string. This corresponds to setting $f(x) \equiv 0$ and $q \equiv 0$ in the previous equations.

\section{A. Vacuum solution}

For the case of the pure gauge string, there is a vacuum solution of the equations. Setting $P(x)=0$ and $h(x)=1$, we find from (19), that:

$$
N^{2}(x) L(x)=A \sin (\sqrt{3 \bar{\Lambda}} x)+B \cos (\sqrt{3 \bar{\Lambda}} x), \quad A, B \text { constants . }
$$

Using the boundary conditions (21), we find the following solution:

$$
N^{2}(x) L(x)=\frac{1}{\sqrt{3 \bar{\Lambda}}} \sin (\sqrt{3 \bar{\Lambda}} x) .
$$

This then can be put into (17) and (18) and we find the solutions:

$$
N(x)=\cos ^{2 / 3}\left(\sqrt{3 \bar{\Lambda}} \frac{x}{2}\right)
$$

and

$$
L(x)=\frac{2^{2 / 3}}{\sqrt{3 \bar{\Lambda}}}[\sin (\sqrt{3 \bar{\Lambda}} x)]^{1 / 3}\left[\tan \left(\sqrt{3 \bar{\Lambda}} \frac{x}{2}\right)\right]^{2 / 3}
$$

where the coefficients again result from the boundary conditions (21). The first zero of $N(x)$ lies at $x_{0}^{v}=\pi / \sqrt{3 \bar{\Lambda}}$. At the same time, $L\left(x \rightarrow x_{0}^{v}\right) \rightarrow \infty$. This is the cosmological horizon of the vacuum solution. If we expand the metric functions around this horizon, we obtain:

$$
N\left(x \rightarrow x_{0}^{v}\right) \approx\left(-\frac{\sqrt{3 \bar{\Lambda}}}{2}\right)^{2 / 3}\left(x-x_{0}^{v}\right)^{2 / 3}+\ldots
$$

and 


$$
L\left(x \rightarrow x_{0}^{v}\right) \approx\left(\frac{\sqrt{3 \bar{\Lambda}}}{2}\right)^{-4 / 3}\left(x-x_{0}^{v}\right)^{-1 / 3}+. .
$$

This has the behaviour of a so-called Kasner solution [20]:

$$
d s^{2}=(k \rho)^{2 a} d t^{2}-d \rho^{2}-C^{2}(k \rho)^{2(b-1)} \rho^{2} d \varphi^{2}-(k \rho)^{2 c} d z^{2}
$$

with $a=c=2 / 3, b=-1 / 3, k=\frac{\sqrt{3 \Lambda}}{2}, C=1$. These type of "closed" solutions have been found previously [5] for the case $\bar{\Lambda}=0$ and were called "supermassive" strings. When

calculating the Kretschmann scalar $K=R^{\mu \nu \rho \sigma} R_{\mu \nu \rho \sigma}$ one obtains [5] that $K \propto\left(x-x_{0}^{v}\right)^{-4}$ and thus the solution has indeed a curvature singularity at $x=x_{0}^{v}$. Remarkable is that in the case of $\bar{\Lambda}=0$, these type of solutions only appear for sufficiently high vacuum expectation values (vev) of the Higgs field corresponding to strings having formed at energy scales much higher than the GUT scale [5]. For smaller values of the vev no singularity appears and the solutions exist on the full interval $[0: \infty$ [. Accordingly, the numerical study showed [7] that these solutions exist for a $\gamma>\gamma_{c r}$.

\section{B. Numerical results}

Subject to the boundary conditions (21), we have studied the coupled system of equations numerically.

First, we fixed $\alpha$ and $\bar{\Lambda}$ to study the influence of the gravitational coupling $\gamma$ on the solutions. We determined the value of $x$ at which the metric function $N(x)$ vanishes, i.e. $N\left(x=x_{0}\right)=0$. Our results for $\alpha=1.0, \bar{\Lambda}=0.005$ and $n=1,2$ are shown in Fig. 1. As expected the value of $x_{0}$ decreases with increasing gravitational coupling $\gamma$. Moreover, we observe a steep decrease in $x_{0}$ for a relatively small range of $\gamma$. We have only plotted results for $\gamma$ 's corresponding to $x_{0} \geq 5$ since for large $\gamma$ 's the numerics becomes increasingly difficult. The reason for this is indicated in Fig. 2, where we show the profiles of the metric functions $N(x), L(x)$ as well as those of the matter field functions $P(x)$ and $h(x)$ for $\alpha=1.0$, $\bar{\Lambda}=0.005, n=1$ and two different choices of $\gamma$. For $\gamma=1.5$, the value of $x$ at which the matter field functions reach their asymptotic values 0 and 1, respectively, is much smaller than the value of $x_{0}$. This means that the horizon clearly lies outside the core of the string. For $\gamma=1.7$, however, the situation is different. The value of $x$ at which $h(x)$ reaches 1 is roughly equal to $x_{0}$, while $P(x)$ seems to be still greater than 0 on the plot we present. This is due to the fact that the "real" solution would have a slightly higher $x_{0}$ at which $P\left(x=x_{0}\right)=0$. However, since $L\left(x \rightarrow x_{0}\right) \rightarrow \infty$, it is numerically impossible to reach the final solution. Nevertheless, the plot indicates that -like $h(x)-P(x)$ just reaches its asymptotic value 0 at $x=x_{0}$. Thus the horizon lies very close to the core of the string.

Then, we fixed $\alpha$ and $\gamma$ and determined $x_{0}$ in dependence on $\bar{\Lambda}$. Our results for $\alpha=$ $\gamma=0.5$ and $n=1,2$ together with the location of the cosmological horizon of the vacuum solution, $x_{0}^{v}$, are given in Fig. 3 .

We clearly observe that the value of the cosmological horizon decreases with the increase of the cosmological constant, as expected. Moreover, an increase in the vorticity $n$ leads to a decrease of $x_{0}$ for the same $\bar{\Lambda}$. For small $\bar{\Lambda}, x_{0}$ of both the $n=1$ and the $n=2$ solution is very close to the corresponding $x_{0}^{v}$. This can again be explained by studying the behaviour of the matter field functions for varying $\bar{\Lambda}$. As observed previously [16], we find that for 
fixed $\alpha$ and $\gamma$ and increasing $\bar{\Lambda}$, the value of the coordinate $x$ at which the matter field functions reach their asymptotic values also increases, e.g. for $\alpha=\gamma=0.5, n=2$ we find that for $\bar{\Lambda}=0.001$ the value of $x$, where the gauge field function reaches $P\left(x^{0.1}\right)=0.1$ is $x^{0.1}(\bar{\Lambda}=0.001) \approx 6.2$, while for $\bar{\Lambda}=0.005$, we find $x^{0.1}(\bar{\Lambda}=0.005) \approx 6.45$. This can be interpreted as representing a thicker string core due to an increased cosmological expansion. Thus the cosmological horizon lies closer and closer to the core of the string for increasing $\bar{\Lambda}$ and so only for small $\bar{\Lambda}$ the solution close to the cosmological horizon can be described by the vacuum solution. In Fig. 4, we show the profiles of $N(x)$ and $L(x)$ for $\bar{\Lambda}=0.01$, $\alpha=\gamma=0.5$ and $n=2$ both for the Nielsen-Olesen string and the vacuum solution. Clearly, the solutions differ quite strongly.

\section{GLOBAL STRINGS IN DE SITTER SPACE}

This is the case of setting $P(x) \equiv n$ and $h(x) \equiv 0$ (which implies $\eta_{1} \equiv 0$ ). The global string without a cosmological constant, i.e. $\bar{\Lambda}=0$ has been studied extensively [8-11]. To have a good starting solution for the construction of dS global strings, we have reconstructed the global string without cosmological constant. We find that the metric function $N(x)$ deviates very little from one, but that the quantity $\left|N(x=0)-N\left(x^{*}\right)\right|$ is increasing with $x^{*} \rightarrow \infty$. Moreover, $L(x)$ grows approximately linearly with $x$. This is the behaviour found in [9], namely that outside the core of the global string the metric functions behave like $N^{2}=1-\frac{\gamma}{2} \ln \left(\frac{x}{x_{g c}}\right), L^{2}=x^{2}\left(1-\gamma \ln \left(\frac{x}{x_{g c}}\right)\right)$ with $x_{g c} \propto\left(q \beta_{2}\right)^{-1}$ being the core of the global string in our rescaled coordinates. Moreover, we recover the behaviour of the Goldstone field function $f(x \rightarrow \infty)=q-O\left(x^{-2}\right)$.

\section{A. Numerical results}

For non-vanishing cosmological constant we find that the behaviour of $N(x)$ and $L(x)$ resembles that of the metric functions in the case of the Nielsen-Olesen string in de Sitter space (see previous section). Again, for all constants fixed and $\bar{\Lambda}$ varied, we find that the value at which $N\left(x=x_{0}\right)=0$ decreases with increasing $\bar{\Lambda}$, e.g. for $\beta_{2}=1, q=0.1, \gamma=0.1$, we find that $x_{0}\left(\bar{\Lambda}=10^{-4}\right) \approx 180$, while $x_{0}\left(\bar{\Lambda}=10^{-3}\right) \approx 57$. At the same time, the value of $x$ at which the function $f(x)$ reaches its vev increases with increasing $\bar{\Lambda}$ which is due to the increased cosmological expansion thus leading to an extended string core. The behaviour of the function $f(x)$ depends crucially on the cosmological constant which determines the cosmological horizon and the parameters $\beta_{2}$ and $q$, which determine the radius of the string core. We will discuss these features in more detail in the context of composite topological defects in the next section.

\section{COMPOSITE SYSTEM OF GLOBAL AND NIELSEN-OLESEN STRING}

Unlike in the case of the "pure" Nielsen-Olesen string, a complete analytical analysis of the composite system seems to be impossible. However, some additional information can be gained by analysing the energy density per unit length. Before discussing our numerical results, we make some remarks on this point in the following section. 
The energy density per unit length of the composite defect is given by $[6,7]$ :

$$
\mathcal{E}=\int \sqrt{-g_{3}} T_{0}^{0} d x_{1} d x_{2}
$$

where $g_{3}$ is the determinant of the $2+1$ dimensional metric $d s^{2}=N^{2}(\rho) d t^{2}-d \rho^{2}-L^{2}(\rho) d \varphi^{2}$ and $T_{0}^{0}=-\mathcal{L}_{M}$ is the 00-component of the energy-momentum tensor. In the cylindrical coordinates, we get:

$$
\begin{aligned}
\mathcal{E} & =\pi \eta_{1}^{2} \int_{0}^{x_{0}} d x N(x) L(x)\left[\frac{n^{2}}{\alpha L^{2}(x)}\left(P^{\prime}(x)\right)^{2}+\left(h^{\prime}(x)\right)^{2}+\left(f^{\prime}(x)\right)^{2}\right. \\
& +\frac{n^{2} h^{2}(x) P^{2}(x)}{L^{2}(x)}+\frac{m^{2} f^{2}(x)}{L^{2}(x)}+\frac{1}{2}\left(h^{2}(x)-1\right)^{2}+\frac{\beta_{2}^{2}}{2}\left(f^{2}(x)-q^{2}\right)^{2} \\
& \left.+\beta_{3}^{2}\left(h(x)^{2}-1\right)\left(f^{2}(x)-q^{2}\right)\right] .
\end{aligned}
$$

From this, we can observe that the composite defect has a finite energy density. There are two different scenarios now. If the product $\beta_{2} q$ is large enough the core of the global string is small and the cores of both strings lie within the cosmological horizon $x_{0}$. We can then assume that $f\left(x_{0}\right)=q, f^{\prime}\left(x_{0}\right)=0, P\left(x_{0}\right)=0, h\left(x_{0}\right)=1$. From before, we know that $L(x)$ goes to infinity close to the horizon, while $N(x) L(x)$ remains finite. Thus the integrand of (31) tends to zero like $m^{2} q^{2} \frac{N(x)}{L(x)}$. If the product $\beta_{2} q$ is small, the core of the global string extends to outside the horizon $x_{0}$. Then the integrand of (31) becomes $N(x) L(x)\left[\left(f^{\prime}(x)\right)^{2}+\frac{m^{2} f^{2}(x)}{L^{2}(x)}+\frac{\beta_{2}^{2}}{2}\left(f^{2}(x)-q^{2}\right)^{2}\right]$. Since close to the horizon, the product $N(x) L(x)$ tends to zero, this is finite. We have indeed confirmed numerically that this is the case.

\section{A. Numerical Results}

Assuming that the behaviour of the metric functions $N(x)$ and $L(x)$ persists in the presence of all (non-trivial) matter fields (which indeed our numerical analysis confirms), we can insert (25) and (26) into (14). We obtain that:

$$
f\left(x \rightarrow x_{0}\right) \sim q+C\left(x-x_{0}\right)^{8 / 3} \quad, \quad C \text { constant }
$$

We have construct the composite model solution by starting from the Nielsen-Olesen (NO) string and increasing the parameter $q$ gradually from zero. Our numerical analysis confirms the assumption that the radius of the NO string core is smaller than that of the global string. Moreover, we find that the NO string always resides inside the horizon. As for the global string, we find that when the coupling constant $\beta_{2}$ is small the function $f(x)$ reaches the imposed expectation value $f\left(x_{0}\right)=q$ with a positive concavity, in particular the derivative $\left.f^{\prime}\right|_{x=x_{0}}$ is non zero and our numerical solution clearly suggests that the behaviour $f\left(x \rightarrow x_{0}\right) \sim q+C\left(x-x_{0}\right)^{8 / 3}$ is not fulfilled. This is demonstrated in Fig. 5, where we show $f(x)$ of the composite defect for $\beta_{2}^{2}=1, \alpha=\gamma=\beta_{1}^{2}=1, q=0.1, \beta_{3}^{2}=0$ and $\bar{\Lambda}=0.001$. This suggests that for small values of the product $\beta_{2} q$, the argument demonstrated above doesn't hold. 
Increasing the value of $\beta_{2}$ we were able to produce solutions which seem to have the expected behaviour, i.e. $f(0)=0, f\left(x=x_{0}\right)=q$ and $\left.f^{\prime}\right|_{x=x_{0}}=0$. This is shown in Fig. 5 for $\alpha=\gamma=\beta_{1}^{2}=1, q=0.1, \beta_{3}^{2}=0, \bar{\Lambda}=0.001$ and $\beta_{2}^{2}=5,10,20,50$, respectively. Of course, the occurrence of the singularity at $x=x_{0}$ renders the interpretation of the numerical results not hundred percent certain but we are rather confident that composite string defects which are regular inside the horizon exist for large enough $\beta_{2}$.

All these results are obtained for the case of $\beta_{3}=0$, i.e. the two defects interact with each other only indirectly over gravity. We have also attempted to construct solutions with $\beta_{3} \neq 0$. We find that only for large enough values of the quotient $\beta_{2}^{2} / \beta_{3}^{2}$, the solutions seem well behaved. If $\beta_{2}^{2} / \beta_{3}^{2}$ is roughly of the order of $10^{2}$ (for $\alpha=\gamma=\beta_{1}^{2}=1, q=0.1$ and $\bar{\Lambda}=0.001)$, the behaviour of the functions is very similar to that in the case of $\beta_{3}=0$. For $\beta_{2}^{2} / \beta_{3}^{2}$ smaller than that, however, the functions $f(x)$ and $h(x)$ start to develop oscillations close to the cosmological horizon. The number of oscillations increases with the decrease of the quotient $\beta_{2}^{2} / \beta_{3}^{2}$. Thus, we conclude that directly interacting composite defects without the global string singularity only exist if the self-interaction of the global string is much larger than the interaction between the two defects.

\section{CONCLUSION}

In this paper we have analysed both Nielsen-Olesen and global strings as well as the composite system of both defects in de Sitter space. When the matter fields are set equal to their vacuum expectation values (vev) in the case of the "pure" Nielsen-Olesen string, we were able to construct analytic solutions of the Einstein equations in terms of trigonometric functions. The metric tensor resembles that of so-called "supermassive" strings which exist in asymptotically flat space only for sufficiently high enough values of the vev of the Higgs field [5]. These were considered as being "unphysical" since they should have formed at energy scales high above the GUT scale. Since recent observations indicate that we live in a universe with positive cosmological constant, and since we find that the existence of our solutions is not restricted to values of the Higgs field's vev being large enough, these solutions might well be of relevance.

Our numerical analysis suggests that the general behaviour of the vacuum metric persists in the presence of the matter fields. E.g. comparing the location of the cosmological horizon $x_{0}$ in dependence on the coupling constants for the "pure" Nielsen-Olesen string and that of the vacuum solution, $x_{0}^{v}$, we find that for small $\bar{\Lambda}$ and/or $\gamma, x_{0}$ and $x_{0}^{v}$ are nearly equal. For increasing $\bar{\Lambda}$ and/or $\gamma$, the difference between the two increases. The reason for this is that the radius of the string core becomes comparable to the radius of the cosmological horizon and thus the assumption of a "vacuum" at the cosmological horizon is not valid any longer.

Constructing the global string and the composite defect of Nielsen-Olesen and global string, we find that the existence of solutions without a singularity resulting from the global string itself depends crucially on the product $\beta_{2} q$ which (in our rescaled coordinates) is inverse proportional to the radius of the global string's core. If (for fixed $q$ ) $\beta_{2}$ is too small, the core of the global string extends to outside the cosmological horizon and the function $f$ reaches its vev with positive concavity. For large enough values of $\beta_{2}$, the core of the global string lies inside the horizon and our numerical results seem to indicate that a global

string/composite defect without the normal singularity of the global string exists. However, 
note that the removal of the global string singularity which exists in asymptotically flat space can be achieved only by introducing a "new" singularity, the curvature singularity at the horizon.

Acknowledgement YB acknowledges the Belgian FNRS for financial support. BH was supported by an EPSRC grant.

Note added After finishing the manuscript, B. Linet has brought to our intention his paper "The static, cylindrically symmetric strings in general relativity with cosmological constant" [J. Math. Phys. 27 (1986), pp. 1817-1818], in which he discusses the vacuum solutions (25) and (26) "re-found" by us. 


\section{REFERENCES}

[1] A. Vilenkin and E. P. S. Shellard, Cosmic strings and other topological defects, Cambridge University Press, Cambridge, England, 1994.

[2] T. W. B. Kibble and M. Hindmarsh, Rep. Progr. Phys. 58, 477 (1995).

[3] H. B. Nielsen and P. Olesen, Nucl. Phys. B61, 45 (1973).

[4] D. Garfinkle, Phys. Rev. D32, 1323 (1985).

[5] P. Laguna and D. Garfinkle, Phys. Rev. D40, 1011 (1989).

[6] M. Christensen, A. L. Larsen and Y. Verbin, Phys. Rev. D60, 125012 (1999).

[7] Y. Brihaye and M. Lubo, Phys. Rev. D62, 085004 (2000).

[8] A. G. Cohen and D. B. Kaplan, Phys. Lett. B215, 67 (1988).

[9] D. Harari and P. Sikivie, Phys. Rev. D37, 3438 (1988).

[10] G. W. Gibbons, M. E. Ortiz and F. Ruiz Ruiz, Phys. Rev. D39, 1546 (1989).

[11] R. Gregory, Phys. Lett. B215, 663 (1988).

[12] M. Barriola and A. Vilenkin, Phys. Rev. Lett. 63, 341 (1989); D. Harari and C. Lousto, Phys. Rev. D42, 2626 (1990).

[13] R. Gregory, Phys. Rev. D54, 4955 (1996).

[14] S. Perlmutter et al., Astrophys. J. 517, 565 (1999); A. G. Riess et al., Astron. J. 116, 1009 (1998).

[15] A. Strominger, JHEP 0110, 034 (2001); JHEP 0111, 049 (2001).

[16] A. M. Gezelbash and R. B. Mann, Phys. Lett B537, 329 (2002).

[17] E. R. Bezerra de Mello, Y. Brihaye and B. Hartmann, Phys. Rev. D67, 045015 (2003).

[18] Y. Brihaye and B. Hartmann, Phys. Rev. D66, 064018 (2002).

[19] J. Spinelly, U de Freitas and E. R. Bezerra de Mello, Phys. Rev. D66, 024018 (2002).

[20] D. Kramer, H. Stephani, E. Herlt and M. MacCallum, Exact solutions of Einstein's field equations, Cambridge University Press, Cambridge, England, 1980. 


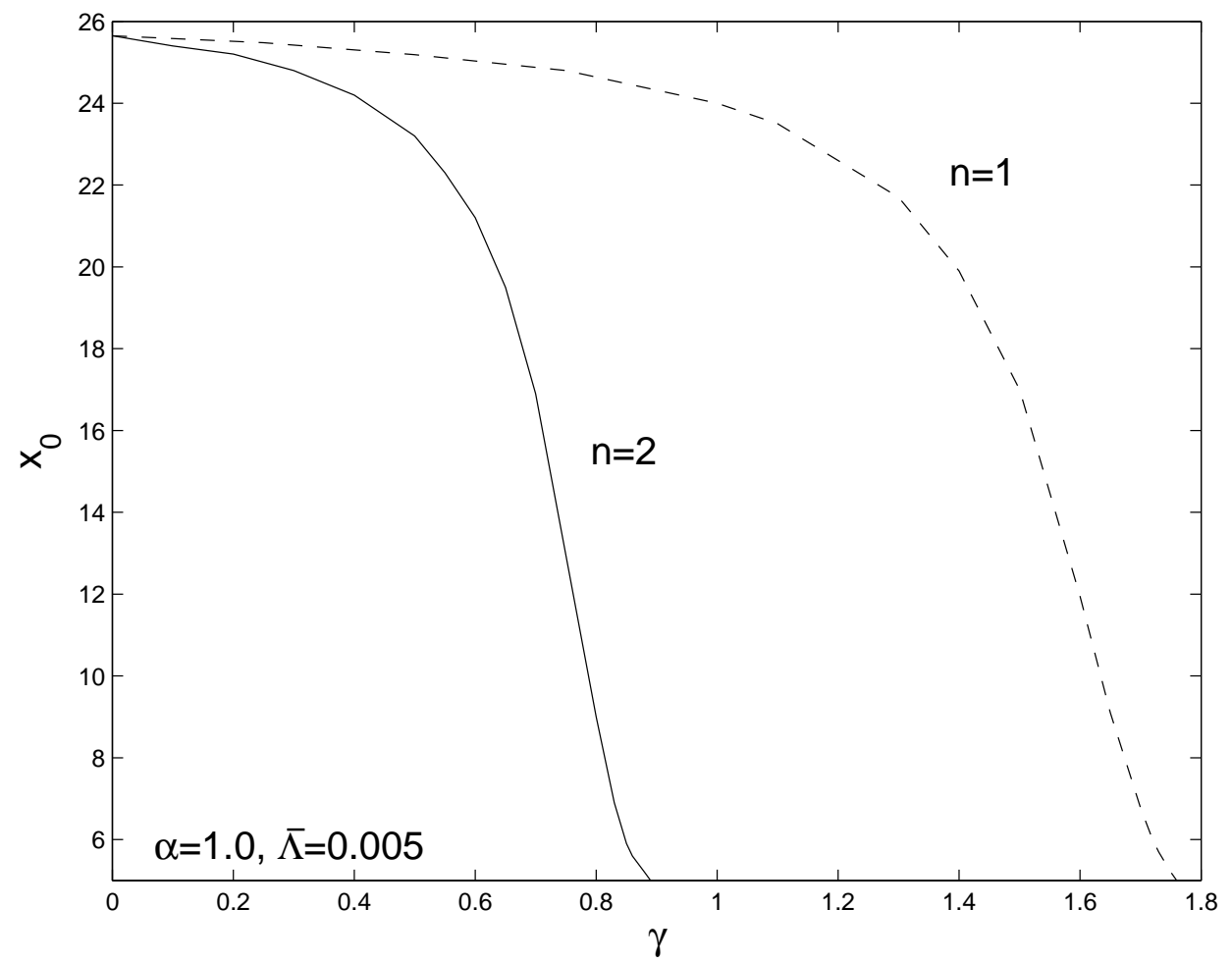

FIG. 1. The value of the dimensionless coordinate $x$ at which a cosmological horizon appears, $x=x_{0}$, is given for the Nielsen-Olesen string as function of $\gamma$ for $n=1$ (dashed) and $n=2$ (solid) with $\alpha=1.0$ and $\bar{\Lambda}=0.005$.

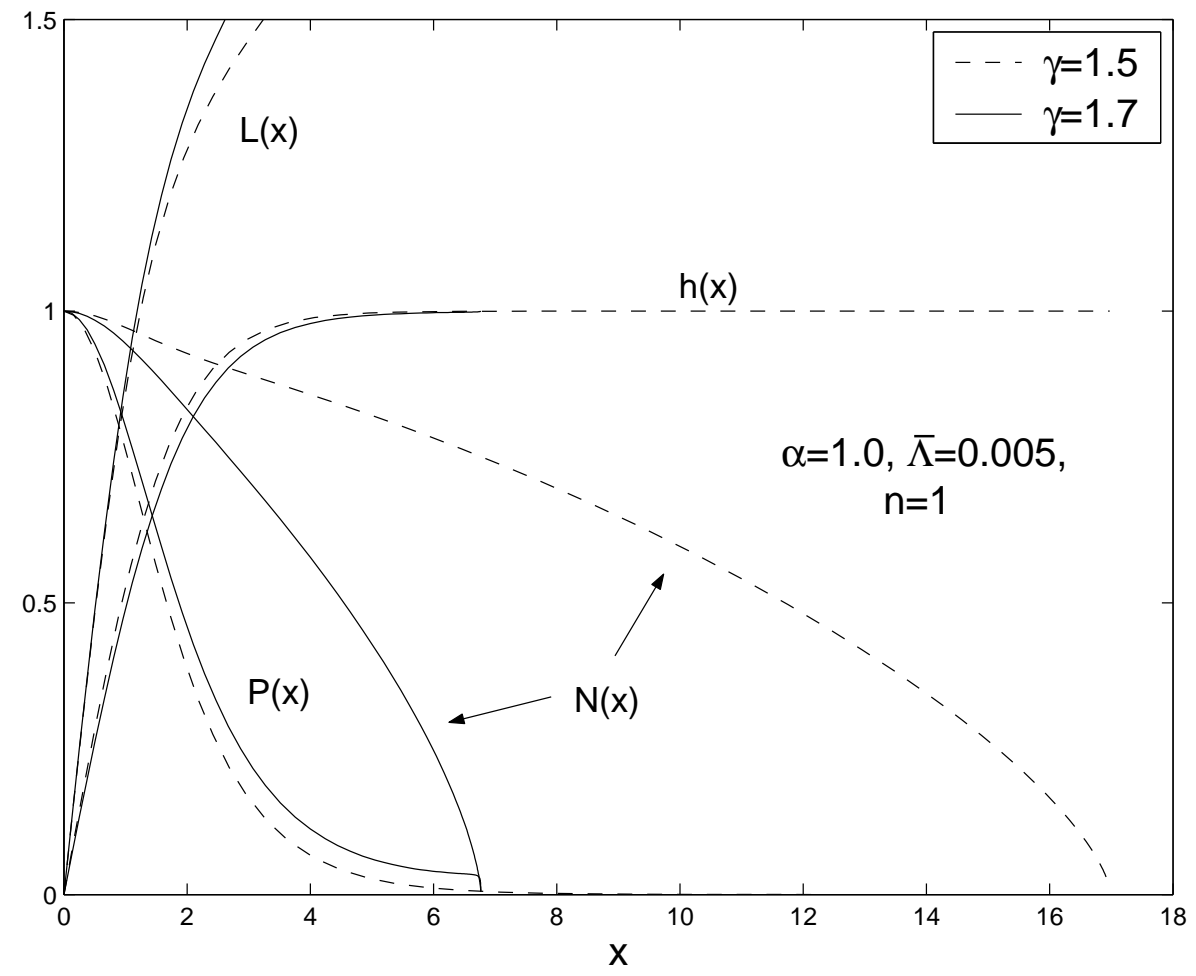

FIG. 2. The profiles of the metric functions $N(x), L(x)$ and the profiles of the matter field functions $P(x)$ and $h(x)$ are shown for $\alpha=1.0, \bar{\Lambda}=0.005, n=1$ and $\gamma=1.5$ (dashed) and $\gamma=1.7$ (solid), respectively. 


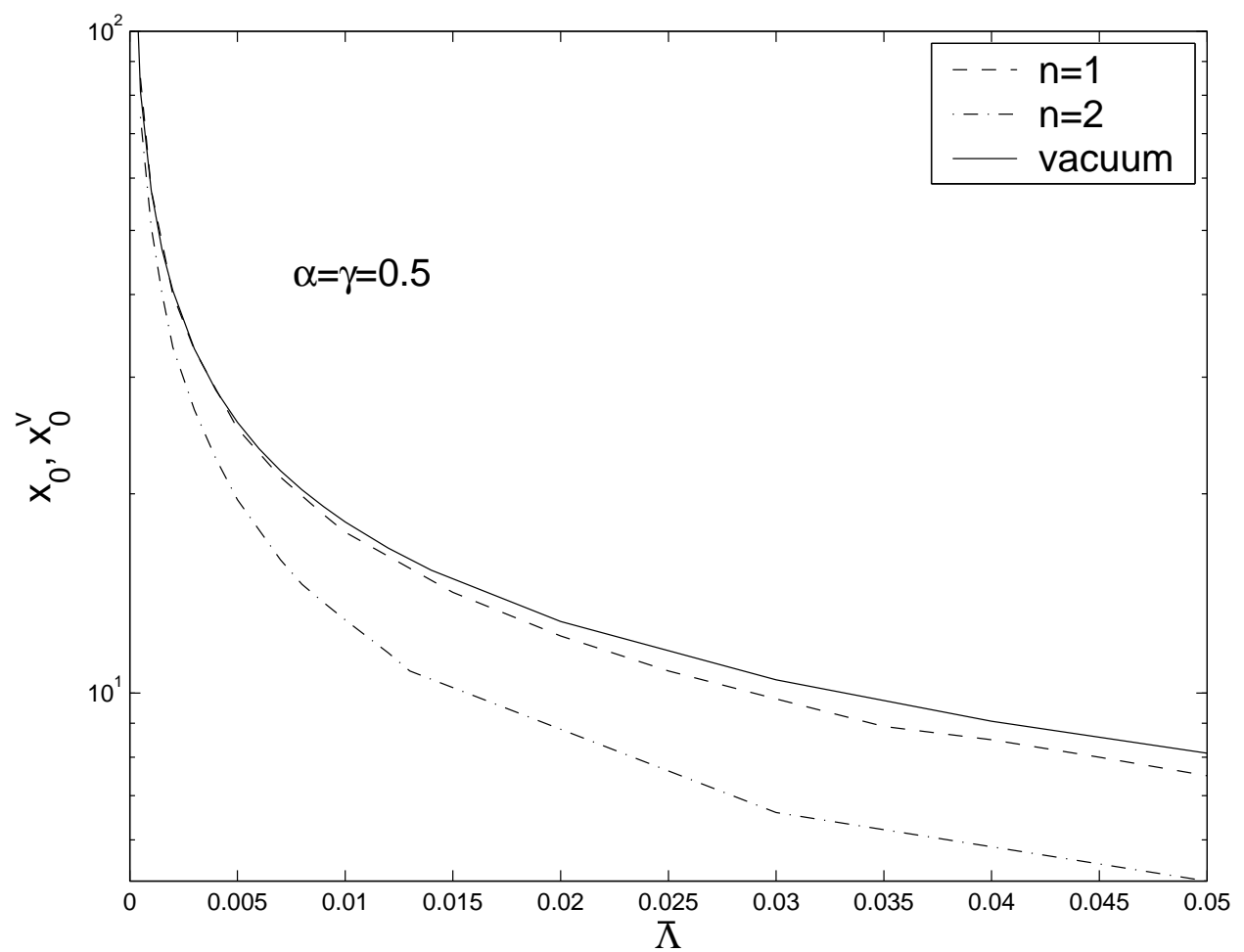

FIG. 3. The value of the dimensionless coordinate $x$ at which a cosmological horizon appears, $x=x_{0}$, is given for the Nielsen-Olesen string as function of $\bar{\Lambda}$ for $n=1$ and $n=2$ with $\alpha=\gamma=0.5$. For comparison, also the value $x_{0}^{v}=\pi / \sqrt{3 \bar{\Lambda}}$ for the vacuum solution is given. 


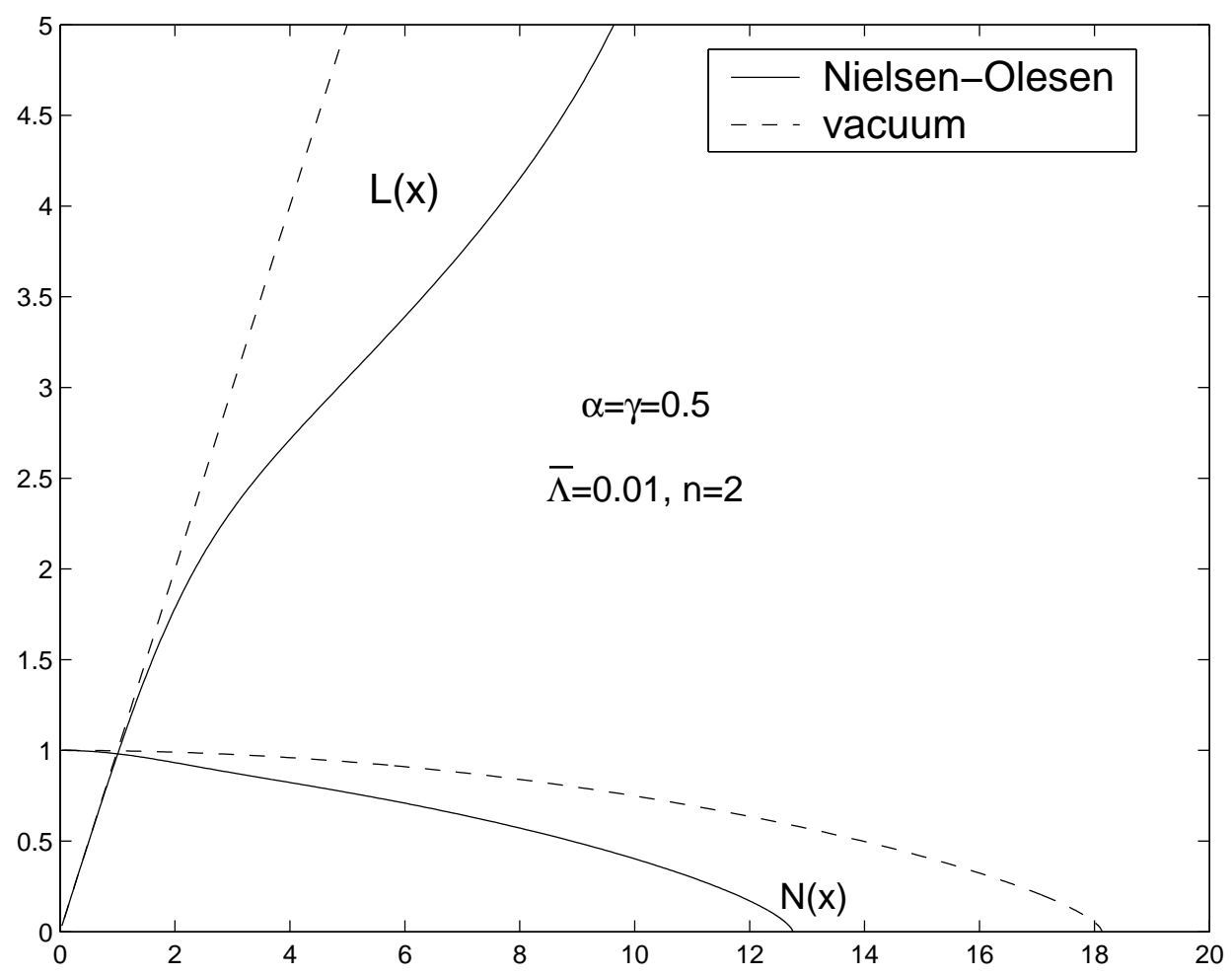

FIG. 4. The profiles of the metric functions $N(x)$ and $L(x)$ are shown for $\alpha=\gamma=0.5, \bar{\Lambda}=0.01$ and vorticity $n=2$. We compare the Nielsen-Olesen solution (solid) with the corresponding vacuum solution (dashed).

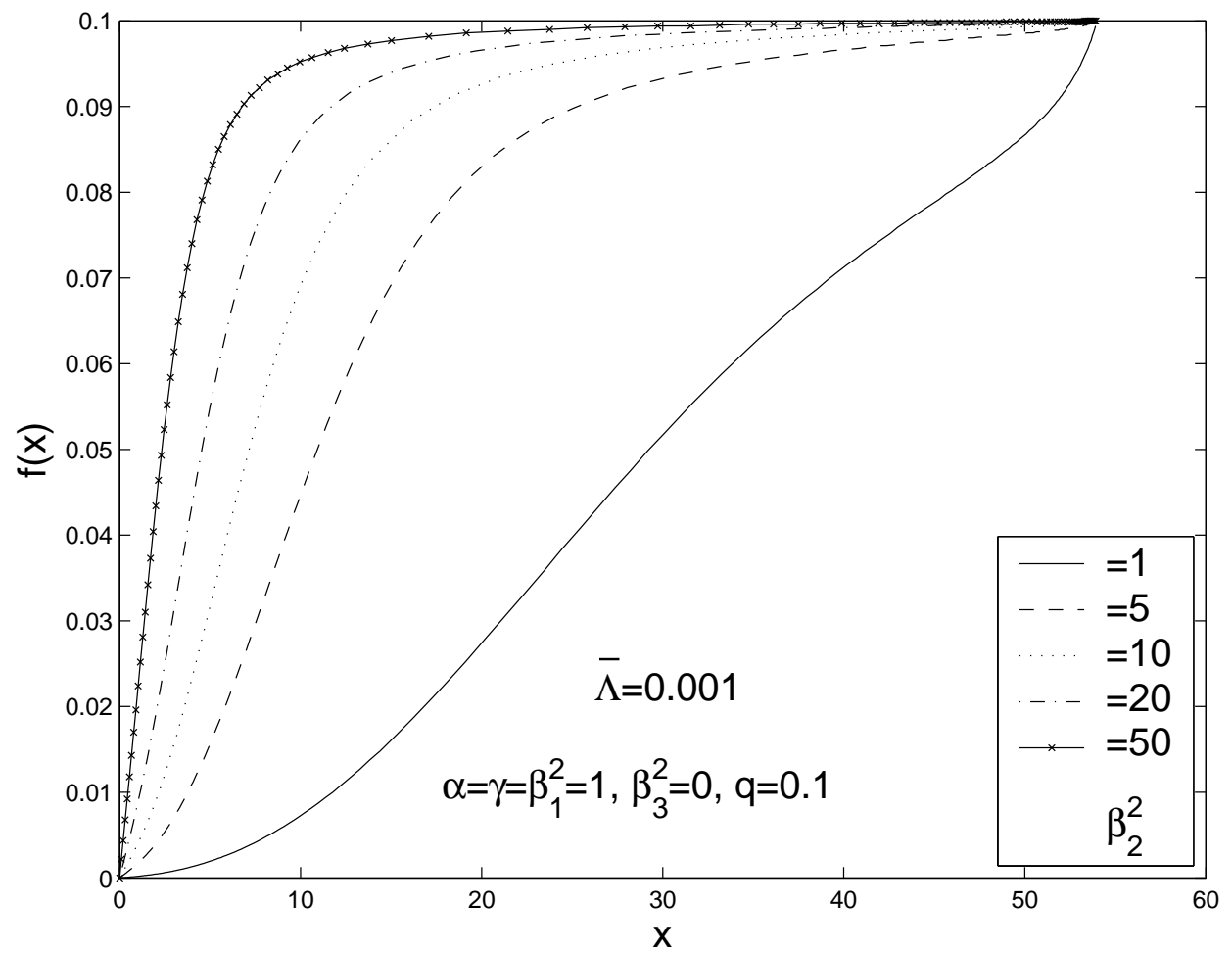

FIG. 5. The profile of the Goldstone field function $f(x)$ is shown for the composite defect with $\alpha=\gamma=\beta_{1}^{2}=1, \beta_{3}^{2}=0, \bar{\Lambda}=0.001, q=0.1$, vorticity $n=1$ and different values of $\beta_{2}^{2}$. 